\title{
Shape optimization of micro-acoustic devices including viscous and thermal losses
}

\author{
Andersen, Peter Risby; Cutanda Henríquez, Vicente; Aage, Niels
}

Published in:

Journal of Sound and Vibration

Link to article, DOI:

10.1016/j.jsv.2019.01.047

Publication date:

2019

Document Version

Peer reviewed version

Link back to DTU Orbit

Citation (APA):

Andersen, P. R., Cutanda Henríquez, V., \& Aage, N. (2019). Shape optimization of micro-acoustic devices including viscous and thermal losses. Journal of Sound and Vibration, 447, 120-136.

https://doi.org/10.1016/j.jsv.2019.01.047

\section{General rights}

Copyright and moral rights for the publications made accessible in the public portal are retained by the authors and/or other copyright owners and it is a condition of accessing publications that users recognise and abide by the legal requirements associated with these rights.

- Users may download and print one copy of any publication from the public portal for the purpose of private study or research.

- You may not further distribute the material or use it for any profit-making activity or commercial gain

- You may freely distribute the URL identifying the publication in the public portal

If you believe that this document breaches copyright please contact us providing details, and we will remove access to the work immediately and investigate your claim 


\section{Accepted Manuscript}

Shape optimization of micro-acoustic devices including viscous and thermal losses

Peter Risby Andersen, Vicente Cutanda Henríquez, Niels Aage

PII: $\quad$ S0022-460X(19)30063-X

DOI: $\quad$ https://doi.org/10.1016/j.jsv.2019.01.047

Reference: $\quad$ YJSVI 14621

To appear in: Journal of Sound and Vibration

Received Date: 28 August 2018

Revised Date: 16 January 2019

Accepted Date: 25 January 2019

Please cite this article as: P.R. Andersen, V.C. Henríquez, N. Aage, Shape optimization of microacoustic devices including viscous and thermal losses, Journal of Sound and Vibration (2019), doi: https://doi.org/10.1016/j.jsv.2019.01.047.

This is a PDF file of an unedited manuscript that has been accepted for publication. As a service to our customers we are providing this early version of the manuscript. The manuscript will undergo copyediting, typesetting, and review of the resulting proof before it is published in its final form. Please note that during the production process errors may be discovered which could affect the content, and all legal disclaimers that apply to the journal pertain. 


\title{
Shape optimization of micro-acoustic devices including viscous and thermal losses
}

\author{
Peter Risby Andersen ${ }^{\mathrm{a}, \mathrm{b}, *}$, Vicente Cutanda Henríquez ${ }^{\mathrm{a}, \mathrm{b}}$, Niels Aage $^{\mathrm{a}, \mathrm{c}}$ \\ ${ }^{a}$ Centre for Acoustic-Mechanical Micro Systems, Technical University of Denmark \\ Ørsteds Plads, Building 352, DK-2800, Kgs. Lyngby, Denmark \\ ${ }^{b}$ Department of Electrical Engineering \\ ${ }^{c}$ Department of Mechanical Engineering
}

\begin{abstract}
Since the late 1980s, numerical shape optimization has been applied successfully to improve the design and development of novel acoustic devices. Most often, viscous and thermal dissipation effects are neglected in the optimization process, as this is an acceptable assumption in e.g. room acoustics, etc. However, in many acoustic devices, ranging from hearing aids to metamaterials, dissipation can significantly influence the acoustic wave behavior. In this paper, we propose a numerical acoustic shape optimization technique and we demonstrate it using two-dimensional quarter-wave and Helmholtz resonators including accurate modelling of viscous and thermal dissipation. By combining a dissipative boundary element method with shape optimization, the sound absorption capability of the resonators located at an impedance tube termination is maximized. Numerical experiments demonstrate the importance of viscothermal dissipation and its impact on the optimization outcome. The resulting resonator shapes, optimized using a lossy assumption, yield significantly better performance compared to their lossless counterpart, with near-perfect absorption at the desired optimization frequencies.
\end{abstract}

Keywords: acoustics, shape optimization, viscothermal losses, boundary element method

\footnotetext{
${ }^{*}$ Corresponding author

Email addresses: prand@elektro.dtu.dk (Peter Risby Andersen), vcuhe@elektro.dtu.dk (Vicente Cutanda Henríquez), naage@mek.dtu.dk (Niels Aage)
} 


\section{Introduction}

Accurate modelling of acoustic devices with small dimensions requires the inclusion of viscous and thermal dissipative effects. The dissipative effects are especially dominant near boundaries, within the viscous and thermal boundary layers, which are in the order of 550-15 $\mu \mathrm{m}$ for air in the audible range. Therefore, as the size of the acoustic domain decreases, the portion of the domain covered by the boundary layers increases, leading to changes in acoustic wave propagation. This situation is very common in small acoustic devices, such as hearing aids, classic and MEMS condenser microphones, where the dimensions are comparable to the thickness of the boundary layers. In such cases, correct modelling of acoustic dissipation is necessary, making the assumption of isentropic wave propagation insufficient [1, 2, 3, 4, 5, 6. The compression driver, usually employed in horn loudspeakers, is also influenced by dissipation in its narrow channels and chambers [7, 8, 9]. Another research field that has received attention in recent years is acoustic metamaterials, where unusual bulk behavior and extraordinary wave propagation effects can be achieved[10, 11]. Recent research has shown that ignoring losses in the design of metamaterials may lead to a degraded behaviour [12, 13. Other publications propose metamaterial designs which take advantage of viscothermal dissipation [14, 15.

Even within the field of room acoustics, micro structures have found their everyday use. For example, the performance of absorbing panels, realized using micro-perforated plates, quarter-wave or Helmholtz resonators, is often very dependent on viscothermal dissipative effects [16, 17.

The importance of the effect of viscothermal dissipation on acoustic wave propagation is very problem dependent. In devices like the aforementioned condenser microphones, neglecting viscothermal losses would yield wrong estimates of their behaviour. In the particular cases studied in this paper it is shown that losses are crucial for obtaining the desired results.

Numerical shape and topology optimization have proven to be valuable tools 
in the discovery of new and better acoustic designs. In the literature, several numerical acoustic optimization approaches exist, making use of either the Finite Element Method (FEM) or the Boundary Element Method (BEM). Especially, optimization of acoustic horns is well studied [18, 19, 20]. Most of these acoustic optimization procedures are based on an isentropic assumption.

35 However, if the influence of viscous and thermal losses is significant, such optimizations might lead to poorly performing designs. In a recent publication, Christensen 21] proposed a method for acoustic FEM topology optimization including viscothermal losses. By applying the so-called low reduced frequency approximation, the author shows how to optimize the cross section of tubes and slits. While topology optimization has certain advantages compared to, e.g., shape optimization, sometimes yielding non-intuitive designs, viscothermal topology optimization requires a mesh that can resolve the boundary layers in the entire design region. This significantly increases the computational load and as a consequence heavily limits the application to full 3D models.

Efficient and reliable optimization tools that include viscothermal losses can be very beneficial in the design of acoustic devices where losses are relevant, such as those mentioned earlier in the introduction. In this work, we propose a shape optimization technique based on BEM and demonstrate it by designing an anechoic termination of a two-dimensional wave-guide by using quarter-wave and Helmholtz resonators. The absorbing properties of the resonators are optimized, showing how viscous and thermal dissipation alters the final layout of the design. Shape optimization is conducted using a BEM implementation incorporating both viscous and thermal dissipation without any simplification other than linearity and absence of flow 22. BEM with losses only requires discretization of the boundaries, thus avoiding the adaptation of the domain mesh and boundary layer meshing, making it very suitable for shape optimization. The proposed method has no restrictions regarding geometry and can be applied to any viscothermal acoustic problem. The paper is organized as follows; firstly, BEM with losses and the optimization approach are introduced. This is followed by a series of simulations investigating shape optimization of quarter- 
wave and Helmholtz resonators. Finally, the possibilities and limitations of the proposed methodology are discussed.

\section{Boundary Element Method with losses}

Numerical modelling of acoustic losses using e.g. FEM, the Finite Difference Method or the Finite Volume Method, is usually realized by discretization of the linearized time-harmonic Navier-Stokes equations [23]. BEM with losses requires a further reformulation of linearized Navier-Stokes equations using the Kirchhoff decomposition 1, 2, resulting in an equation-set given by [24]

$$
\begin{aligned}
& \Delta p_{a}+k_{a}^{2} p_{a}=0 \\
& \Delta p_{h}+k_{h}^{2} p_{h}=0 \\
& \Delta \vec{v}_{v}+k_{v}^{2} \vec{v}_{v}=\overrightarrow{0} \quad \text { with } \quad \nabla \cdot \vec{v}_{v}=0
\end{aligned}
$$

which consists of three separate equations with the unknown variables being the acoustic pressure $p_{a}$, the thermal pressure $p_{h}$ and the viscous velocity $\vec{v}_{v}$. Similarly, $k_{a}, k_{h}$ and $k_{v}$ are the acoustic, thermal and viscous complex wavenumbers, respectively. Eqs. (1) 3 are formally equal to the Helmholtz equation. The frequency dependent wavenumbers are defined as 24.

$$
\begin{aligned}
k_{a}^{2} & =k^{2}\left(1+i k l_{v h}-k^{2} l_{v h}^{\prime} l_{h}\right)^{-1} \\
k_{h}^{2} & =-\frac{i}{l_{h}} k\left(1-l_{v h}^{\prime} i k\right)^{-1} \\
k_{v}^{2} & =-\frac{i \omega \rho}{\mu}
\end{aligned}
$$

with

$$
l_{h}=\lambda /\left(\rho c C_{p}\right) \quad \text { and } \quad l_{v h}^{\prime}=(\gamma-1)\left(l_{h}-l_{v}\right)
$$

where

$$
l_{v}=\left(\frac{4}{3} \mu+\eta\right) /(\rho c) .
$$

In Eqs. (4 8), $k=\omega / c$ is the isentropic wavenumber, $\omega$ is the angular frequency, $c$ is the speed of sound, $\rho$ is the static density, $\mu$ is the shear viscosity, $\eta$ is the bulk viscosity, $C_{p}$ is the heat capacity at constant pressure and $\gamma$ is the ratio 
of specific heats. We remark that the wavenumbers are based on a second order Taylor approximation as presented in Ref. 24]. Comparative simulations including the full expressions of the wavenumbers have shown no significant variations in the results. The Kirchoff decomposition is convenient because each of the equations can easily be transformed into integral from, so that

$$
\begin{aligned}
C(P) p_{a}(P) & =\int_{\Gamma} \frac{\partial G(R)}{\partial n(Q)} p_{a}(Q) \mathrm{d} \Gamma-\int_{\Gamma} G(R) \frac{\partial p_{a}(Q)}{\partial n(Q)} \mathrm{d} \Gamma \\
C(P) p_{h}(P) & =\int_{\Gamma} \frac{\partial G(R)}{\partial n(Q)} p_{h}(Q) \mathrm{d} \Gamma-\int_{\Gamma} G(R) \frac{\partial p_{h}(Q)}{\partial n(Q)} \mathrm{d} \Gamma \\
C(P) v_{v, x}(P) & =\int_{\Gamma} \frac{\partial G(R)}{\partial n(Q)} v_{v, x}(Q) \mathrm{d} \Gamma-\int_{\Gamma} G(R) \frac{\partial v_{v, x}(Q)}{\partial n(Q)} \mathrm{d} \Gamma \\
C(P) v_{v, y}(P) & =\int_{\Gamma} \frac{\partial G(R)}{\partial n(Q)} v_{v, y}(Q) \mathrm{d} \Gamma-\int_{\Gamma} G(R) \frac{\partial v_{v, y}(Q)}{\partial n(Q)} \mathrm{d} \Gamma
\end{aligned}
$$

with $C(P)$ being the integral-free term, $P$ is the collocation point, $Q$ is an integration point on the generator, $R=|Q-P|$ is the distance between $P$ and $Q$ and $G$ is the fundamental solution in two dimensions. In this case we assume two-dimensional domain, and the viscous velocity $\vec{v}_{v}$ is split into its two Cartesian components $v_{v, x}$ and $v_{v, y}$. Eqs. (9)-12 can then be discretized and coupled at the boundary by applying a no-slip and an isothermal boundary condition 25$]$

$$
\begin{aligned}
& \vec{v}_{b}=\phi_{a} \nabla p_{a}+\phi_{h} \nabla p_{h}+\vec{v}_{v} \\
& T=\tau_{a} p_{a}+\tau_{h} p_{h} \simeq 0
\end{aligned}
$$

where $\phi_{a}, \phi_{h}, \tau_{a}$ and $\tau_{h}$ are complex constants, derived from the physical quantities: the static density, the speed of sound, the ratio of specific heats at constant pressure, the thermal conductivity, the coefficient of viscosity, the bulk viscosity and the frequency. $T$ is the temperature perturbation at the boundary and $\vec{v}_{b}$ is the boundary velocity. The total pressure perturbation is the sum of the acoustic and thermal pressures. After coupling and discretization, the final linear system has the form

$$
\mathbf{S}\left[\begin{array}{c}
\mathbf{p}_{a} \\
\mathbf{v}_{v, x} \\
\mathbf{v}_{v, y}
\end{array}\right]=\left[\begin{array}{c}
\mathbf{v}_{b, n} \\
0 \\
\mathbf{v}_{b, t}
\end{array}\right]
$$


The small bold letters represent the discretized variables and the subindices $n$ and $t$ indicate the boundary normal and tangential components. The matrix $\mathbf{S}$ is the system matrix assembled according to the method found in Ref. 22, with the assumption of two dimensions. Note that $p_{h}$ has been eliminated from the final system by a Schur complement operation.

Because the optimization approach introduced in the following sections requires repeated function evaluations, code efficiency is important. Therefore, assembly of the BEM matrix is carried out using a compiled MATLAB ${ }^{\circledR}$ MEX/C ++ framework, inspired by the BEM software OpenBEM [26]. The C++ implementation has proven to be an order of magnitude faster as compared to the pure MATLAB ${ }^{\circledR}$ OpenBEM code, thus improving the efficiency and usability of the 75 proposed optimization approach.

Additionally, it is possible to take advantage of the sparse nature of the viscous and thermal matrices (the matrices assembled by discretization of Eqs. (10)-(12). An increase of the computational speed is gained by only performing integration when the distance between $\mathrm{P}$ and $\mathrm{Q}$ is less than twenty times the boundary layer thickness, or if $\mathrm{P}$ and $\mathrm{Q}$ are located on the same element. This threshold is considered sufficient, but could probably be further reduced. The potential errors of performing the truncation during the assembly process are further discussed in Appendix A By using this simple approach an additional order of magnitude in computational speed is achieved when creating the viscous and thermal matrices.

\section{Geometry and parametrization}

In acoustics, characterization of absorbing materials is often realized using the impedance tube method [27. Fig. 1 depicts the two-dimensional computational domain $\Omega$, which can be regarded as conceptually equivalent to a three-dimensional tube. It will be referred to as an impedance tube in the following. At the left-hand boundary, the impedance tube is excited by a normal velocity condition with $v_{b, n}=1 \mathrm{~mm} / \mathrm{s}$, arbitrarily chosen. The impedance tube 


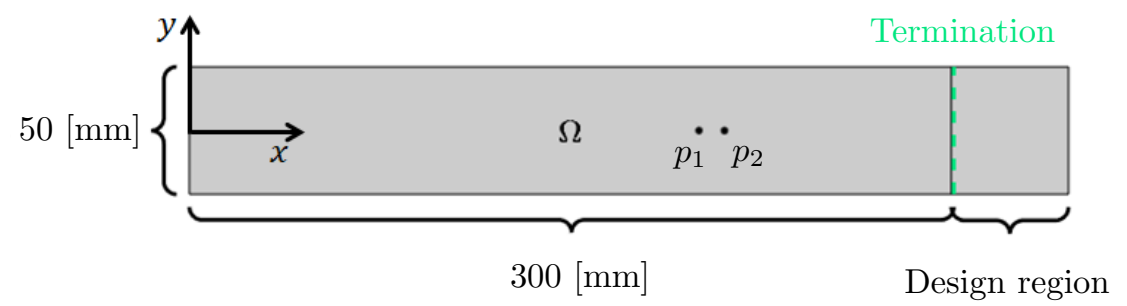

Figure 1: The two-dimensional computational domain $\Omega$ including the design region. The two acoustic pressures at $p_{1}$ and $p_{2}$ are used to calculate the absorption coefficient of the impedance tube termination.

is terminated at the right end by a design zone that is occupied by resonators of either the quarter-wave or Helmholtz types. The resonators are parametrized independently using the same symmetric cubic spline description. Therefore, all resonators have the same shape. This approach was chosen in order to reduce the overall number of design variables. The initial parametrized shape and the locations of the cubic spline control points forming the quarter-wave and Helmholtz resonators are seen in Fig. 2a and Fig. 2b, respectively. The shape of the resonators is controlled by changing the positions of the control points (illustrated by blue dots in Fig. 2). Four additional design parameters are used to allow the resonators to scale up to $10 \%$ in length individually.

In shape optimization, choosing an appropriate parametrization method is often difficult. The cubic spline parametrization proposed in this work provides a degree of boundary regularization, at the cost of restricting the possible boundary shapes. This means, e.g., that the resonators cannot form sharp edges and corners. To ensure maximum design freedom, the control points are allowed to move freely in any direction, with the constraints that boundaries are not allowed to intersect and the maximum curvature is limited. The boundary is discretized using approximately 1400 continuous quadratic isoparametric Lagrange boundary elements. Quadratic elements will help maintain boundary smoothness when distorted. In the majority of the presented results, the 
a)

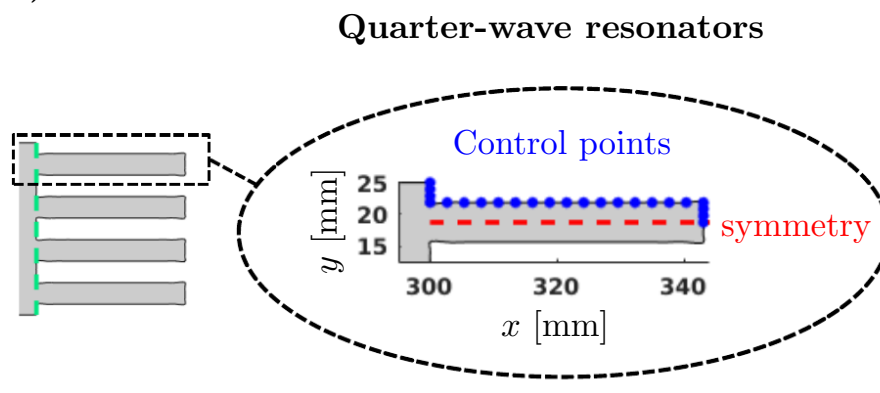

b)

\section{Helmholtz resonators}

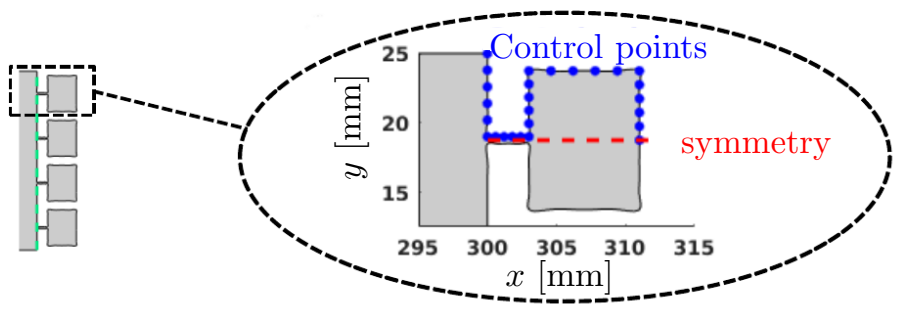

Figure 2: The initial parametrization of the impedance tube termination. The two resonator types used are a) quarter-wave resonators and b) Helmholtz resonators. At the left-hand side of a) and b), the full design is shown. The parametrization of a single resonator is magnified, showing the initial locations of the control points (blue dots) that determine the shape of the resonators.

cubic spline parametrization consists of 23 or 26 control points describing the shape of the quarter-wave or the Helmholtz resonators, respectively. The spline parametrization is implemented using the MATLAB ${ }^{\circledR}$ function cscvn.

\section{Optimization approach}

The shape optimization is performed using the gradient-based sequential quadratic programming approach found in MATLAB ${ }^{\circledR}$ 's fmincon function [28]. 
The optimization problem is given by

$$
\begin{array}{ll}
\max _{\mathbf{v}} & \phi(\mathbf{v})=1-\left|\frac{\mathrm{e}^{-\mathrm{ik}_{\mathrm{LL}} \mathrm{d}}-\frac{\mathrm{p}_{2}(\mathbf{v})}{\mathrm{p}_{1}(\mathbf{v})}}{\frac{\mathrm{p}_{2}(\mathbf{v})}{\mathrm{p}_{1}(\mathbf{v})}-\mathrm{e}^{\mathrm{i} \mathrm{k}_{\mathrm{LL}} \mathrm{d}}}\right|^{2} \\
\text { s. t. } & \boldsymbol{\kappa}(\mathbf{v})-\kappa_{\max } \leq 0 \\
& D_{\min }-\beta \mathbf{D}(\mathbf{v}) \leq 0 .
\end{array}
$$

Eq. 15

where $\mathbf{v}$ are the design variables and $\phi(\mathbf{v})$ is the objective function. The goal is to maximize the absorption coefficient, ranging from 0 (no absorption) to 1 (perfect absorption). The objective function represents therefore the absorption coefficient, obtained using the transfer function method [27. This method only requires the evaluation of the complex pressure at two positions $p_{1}$ and $p_{2}$, located at $x=200 \mathrm{~mm}$ and $x=210 \mathrm{~mm}$, respectively. The location of $p_{1}$ and $p_{2}$ is depicted in Fig. 1. Furthermore, $d=10 \mathrm{~mm}$ is the distance between $p_{1}$ and $p_{2}$ and $k_{L L}$ is the lossless wavenumber. The domain pressures $p_{1}$ and $p_{2}$ are obtained via field point calculations of the corresponding acoustic and thermal pressures, using

$$
C\left(P_{j}\right) p_{a}\left(P_{j}\right)=\int_{\Gamma} \frac{\partial G(R)}{\partial n(Q)} p_{a}(Q) \mathrm{d} \Gamma-\int_{\Gamma} G(R) \frac{\partial p_{a}(Q)}{\partial n(Q)} \mathrm{d} \Gamma
$$

and

$$
C\left(P_{j}\right) p_{h}\left(P_{j}\right)=\int_{\Gamma} \frac{\partial G(R)}{\partial n(Q)} p_{h}(Q) \mathrm{d} \Gamma-\int_{\Gamma} G(R) \frac{\partial p_{h}(Q)}{\partial n(Q)} \mathrm{d} \Gamma
$$

where $P_{j}$ represents the location of either $p_{1}$ or $p_{2}$ and $R=\left|P_{j}-Q\right|$. The total pressure on the two positions is found using that $p=p_{a}+p_{h}$. Note that the state problem is fullfilled at every cycle, i.e., following the so-called nested formulation [29]. The absorption coefficient is a function of the boundary parametrization $\mathbf{v}$, which is a vector containing the coordinates of the control points as well as the scaling factor for the resonator length.

To avoid non-physical boundary behavior, the parametrized boundary is constrained using the curvature magnitude $\boldsymbol{\kappa}$ located at the control points and the distance between control points $\mathbf{D}$ (see Figure 3 ). The vector $\mathbf{D}$ contains all 
a)

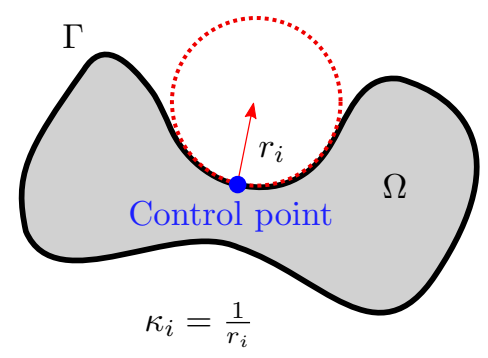

b)

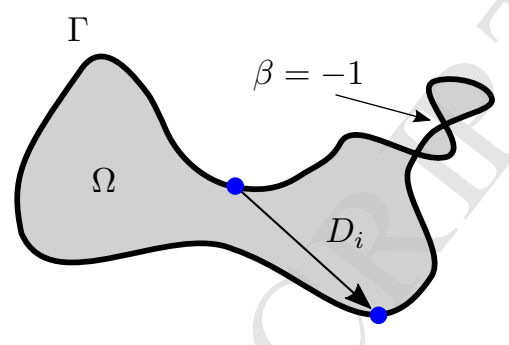

Figure 3: Illustration of the curvature and distance constraints used during optimization. In Figure a) the curvature constraint is illustrated with $\kappa_{i}$ begin the curvature at a control point. Figure b) shows the distance constraint with $D_{i}$ begin the distance between two control points. Moreover, $\beta$ is a parameter that is -1 if the boundary self-intersects and 1 otherwise.

possible combinations of distances between control points, with a single entry in $\mathbf{D}$ defined as

$$
D_{i}=\sqrt{\left(x_{l}-x_{j}\right)^{2}+\left(y_{l}-y_{j}\right)^{2}} .
$$

Here, $x$ and $y$ are the Cartesian coordinates of the control points with the subscripts $l$ and $j$ denoting two different control points. Additionally, we introduce the parameter $\beta$ which equals -1 if the parametrized boundary self-intersects or 1 otherwise. Here, $\beta$ is found by using the implementation for detecting self-intersections found in Ref. [30. In this way, too sharp boundaries and selfintersection are avoided. The maximum allowed curvature at the control points is $\kappa_{\max }=6.3 \mathrm{~mm}^{-1}$ and the minimum distance between the individual control points is $D_{\min }=1 \mu \mathrm{m}$, cf. the dimensions given in Fig 1 . Because the boundary is parametrized using $C^{2}$ continuous cubic splines, the curvature at a control point is easily obtained in a two-dimensional Cartesian coordinate system as 31 .

$$
\kappa_{i}=\frac{\left|x^{\prime}(t) y^{\prime \prime}(t)-y(t)^{\prime} x(t)^{\prime \prime}\right|}{\left(x^{\prime}(t)^{2}+y^{\prime}(t)^{2}\right)^{3 / 2}}
$$

where $x$ and $y$ are the coordinates of a control point as a function of the cubic spline parameter $t \in[0,1]$, with the primes denoting the derivative with respect 
to $t$. The derivatives are evaluated using the MATLAB ${ }^{\circledR}$ functions fnder and fnval. The constraints require additional distance calculations between control points, detection of boundary intersections and curvature calculations, making optimization more computationally demanding. Constraints could be avoided by selecting a more restricted parametrization, that enforces a physical behaviour. This would however limit the possible shapes and design freedom. As a benefit, the constraints can also be used to specify and include manufacturing tolerances in the design process.

Gradient information is obtained using a simple forward finite difference (FD) scheme

$$
\frac{\partial \phi}{\partial v_{i}}=\frac{\phi\left(\mathbf{v}+\Delta v_{i} \cdot \mathbf{e}_{i}\right)-\phi(\mathbf{v})}{\Delta v_{i}}
$$

where $v_{i}$ is a single design variable, $\mathbf{e}_{i}$ is a unit vector for the $\mathrm{i}$-th variable and $\Delta v_{i}$ is the step length evaluated using fmincon's default step length. The choice of the step length is important for the accuracy of the finite difference sensitivities. Therefore, the usage of the default step length is briefly discussed in Appendix B. Efficient adjoint methods can be found in the literature for lossless acoustic BEM shape optimization [32, 18. However, equivalent adjoint methods for lossy BEM are currently not available. To improve the speed of the FD gradient evaluation fmincon's UseParallel option is enabled, allowing the FD gradients to be calculated in parallel. Optimization is performed on a desktop PC with six cores. The FD gradient estimation used is computationally reasonable for the presented case. However, performing optimization of larger problems including more design variables would require an adjoint approach. This is, however, deemed outside the scope of the presented work.

\section{Results}

In the following, we investigate how viscous and thermal damping affect the design obtained by shape optimization for the impedance tube example. In Section 5.1. quarter-wave resonators are optimized using lossless and lossy 
BEM formulations, including single- and multi-frequency optimization. As an additional test, the quarter-wave resonators are optimized using a simple bulk loss model. In the second example in Section 5.2 , the impedance termination is initialized as Helmholtz resonators which are then further optimized.

\subsection{Quarter-wave resonators}

\section{Lossless optimization}
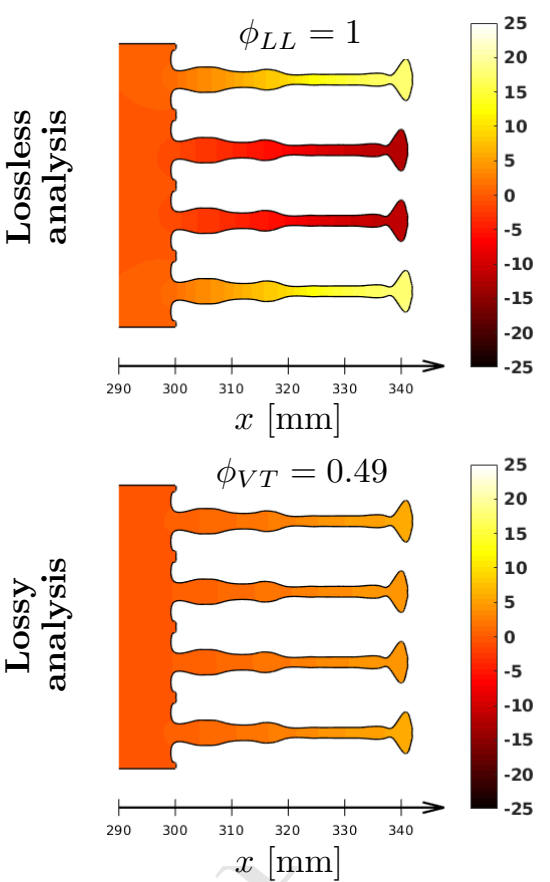

\section{Lossy optimization}
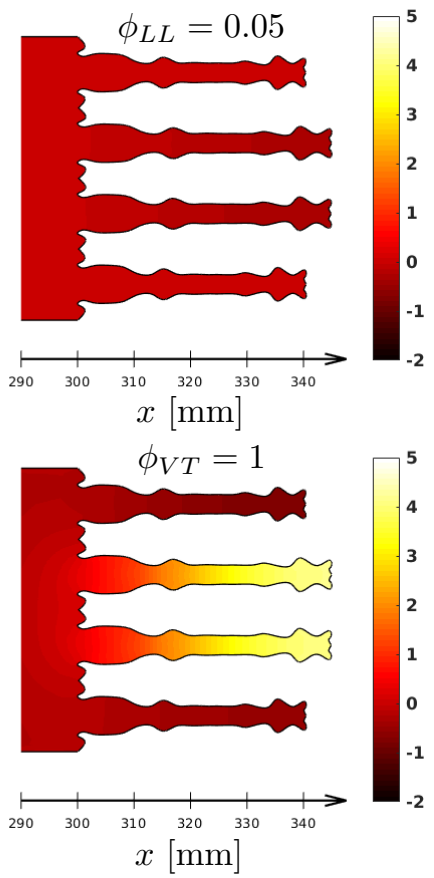

Figure 4: Comparison (crosscheck) of the lossless and lossy optimized designs of the impedance tube termination. Here, $\phi_{L L}$ and $\phi_{V T}$ are the lossless and lossy objective functions calculated for the two designs. The initial design consists of simple quarter wave resonators controlled using a cubic spline representation of the boundary, as depicted in Fig. 2a. The plotted field variable is the real part of the pressure in Pascal.

Fig. 4 shows the result of a single frequency shape optimization for the quarter-wave resonators problem. The resonators are optimized for maximum 

lossy BEM formulations, with $\phi_{L L}$ and $\phi_{V T}$ denoting the objective function of the final design, calculated either using a lossless or lossy assumption, respectively. This makes it possible to analyze how the viscothermal losses will impact the final resonator shape. As seen in Fig 4 the two designs deviate

absorption at $2 \mathrm{kHz}$. The optimization was conducted using both lossless and in shape depending on whether losses are considered in the design (right side plots) or not (left side plots). The lossless design forms narrower resonators and has different features compared to that of the lossy design. This is an interesting result because narrow domain parts should contribute to increased absorption, and here, the narrowest design is achieved assuming no losses. It is worth noting that both designs perform perfectly under the settings for which they are optimized, i.e. $\phi_{L L}=1$ for the lossless design and $\phi_{V T}=1$ for the lossy design. The performance of the lossless model is a particularly surprising result, as the lossless design is not expected to include any dissipation. Hence, it should not be capable of absorbing any sound. The reason for this surprising result is due to a peculiarity of numerical BEM: interior BEM problems are prone to numerical damping as reported in [33, 34, 35. This is most often not a problem when only performing standard analysis of acoustic devices. When optimization is applied using lossless BEM, the optimizer makes use of numerical damping. The design is therefore stable, but unphysical. Had the optimization been performed using a standard FEM model for the analysis, this would not have been the case. Lossless FEM does not introduce any additional phase with respect to the excitation, which translates into a purely imaginary complex pressure. However, in BEM the complex pressure has a real part indicating an additional phase shift due to numerical damping. The real part of the pressure is therefore plotted in Fig 4 as a demonstration. Finally, it is worth noting that while the lossless design does in fact have an absorption coefficient of 0.49 when analysed using the lossy model, the lossy design has almost no absorption capabilities when modelled using the lossless model. This makes it interesting to investigate the influence of the damping mechanism on the optimized designs and also, how much can be achieved using the simple "lossless" BEM model. To 
assist in understanding the damping mechanisms, Fig. 5 shows the magnitude of the thermal pressure and viscous velocity for both lossy and lossless designs evaluated using the visco-thermal acoustic model.

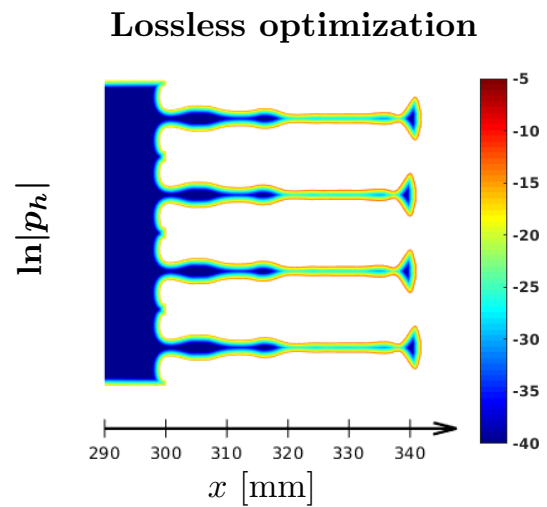

\section{Lossy optimization}
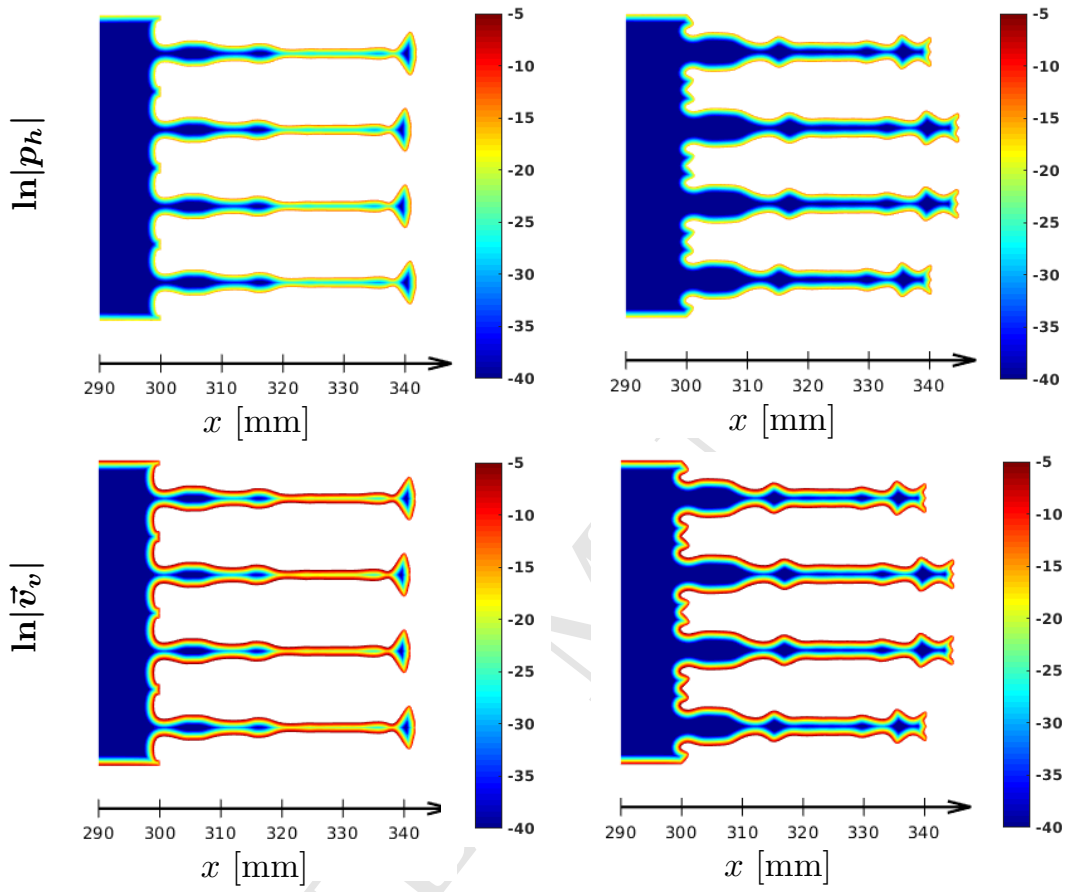

Figure 5: Domain calculations of the thermal pressure and the viscous velocity for the lossless and lossy optimized designs. To enhance the visual representation of field variables, the natural logarithm of the magnitude of the thermal pressure and viscous velocity modulus is plotted in the colormaps.

First, we examine how the numerical damping performs as compared to the true physical viscothermal model. We start by noting that for the lossless BEM model, convergence to full absorption can only be achieved when the tubes are allowed to scale in length individually. This allows the quarter-wave resonators to become slightly de-tuned, making it possible for the acoustic energy to be exchanged between the resonators rather than being reflected. This effect is often 
used in recent publications on metamaterial absorbing surfaces, such as [36, 37. The resonators in the example form two de-tuned pairs, upper and lower, with the same properties. Combining the phenomena of de-tuned resonators with the inherent numerical damping, consequently gives a lossless design that appears to be a perfect absorber. As already mentioned, it is interesting to note that the lossless design contains more narrow tube sections than the lossy design. This leads to a substantial overlap in the boundary layers as seen Fig. 5(left). However, and despite the pronounced overlap, the performance is only at 0.49 , indicating that the overlapping boundary layer is not beneficial. This observation in further backed when inspecting the distribution of thermal and viscous boundary layers for the lossy design.

On the other hand, the lossy design perfectly absorbs sound at the optimization frequency. Here, the thickness of the resonators is larger compared to the lossless design and the entrance of the resonators has a rippled funnel-shape. The entrance presumably acts as an impedance match, so the acoustic wave can ${ }_{225}$ propagate easier into the narrow region where losses are more significant. At the end of the quarter wave resonators, the boundary is slightly expanded, a possible attempt to maximize boundary length in the region where the pressure magnitude is larger, contributing with additional losses. That this is indeed the case, it is further confirmed when inspecting Fig. 5(right) which shows that the channel width never gets so small that the boundary layers overlap, although they almost meet at several positions. That is, for this specific optimization setup the optimization routine seems to prefer a design with compactly placed boundary layers and no boundary layer overlapping to achieve high energy dissipation. The lengths of the resonators are organized in a similar pattern as the lossless design, seemingly taking advantage of the de-tuned resonator phenomenon. However, the length difference between the de-tuned resonators is slightly larger, and the pressure in the tubes is significantly reduced as compared to that of the lossless design.

Fig. 6 shows the evolution of the objective function during the optimization process for both lossless and lossy models, with blue and red curves correspond- 


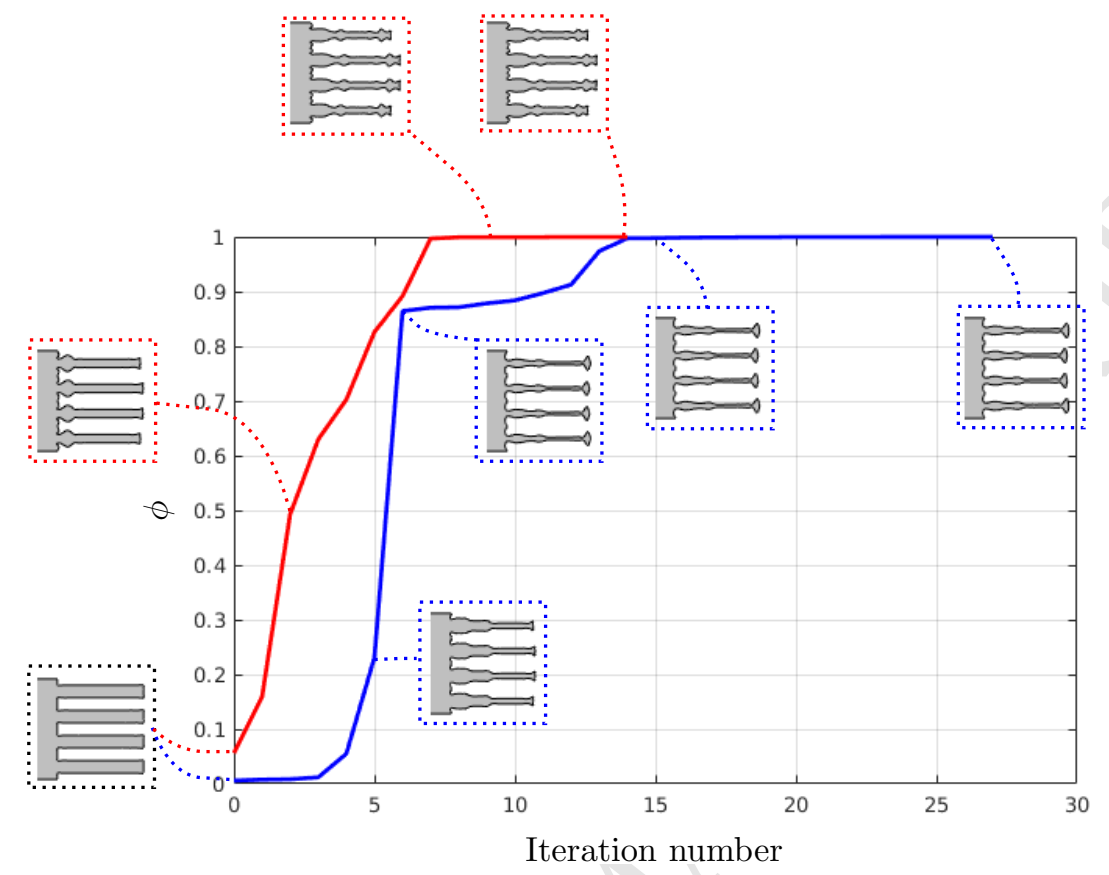

Figure 6: The evolution of the objective function for lossless (blue curve) and lossy (red curve) quarter-wave resonator shape optimization. Selected design shapes obtained at specific iterations are included.

ing to $\phi_{L L}$ or $\phi_{V T}$, respectively. In this example, optimization with losses requires less iterations to achieve near-perfect absorption as compared to lossless optimization. In the lossless case, there is a large change in the objective function between iteration 5 and 6 , which is most likely due to the unstable nature of resonances. Such behaviour is not observed when losses are included. Viscothermal losses stabilize and reduce high-pressure amplitudes near resonance. The total optimization time is $194 \mathrm{~min}$ for the lossless design and 135 min for the lossy design. More importantly, we note that the chosen optimizer solves the chosen problem in Eqs. (16) to stationarity, i.e., to an actual KarushKuhn-Tucker (KKT) point. This is clearly seen in Fig. 6 where the change in objective, as well as the change in layout, is negligible after reaching half of the used iterations. Hence, from an engineering perspective, one could with confidence apply a stopping criteria based on the objective function and the change 
in layout, and thus save $50 \%$ of the computational time.

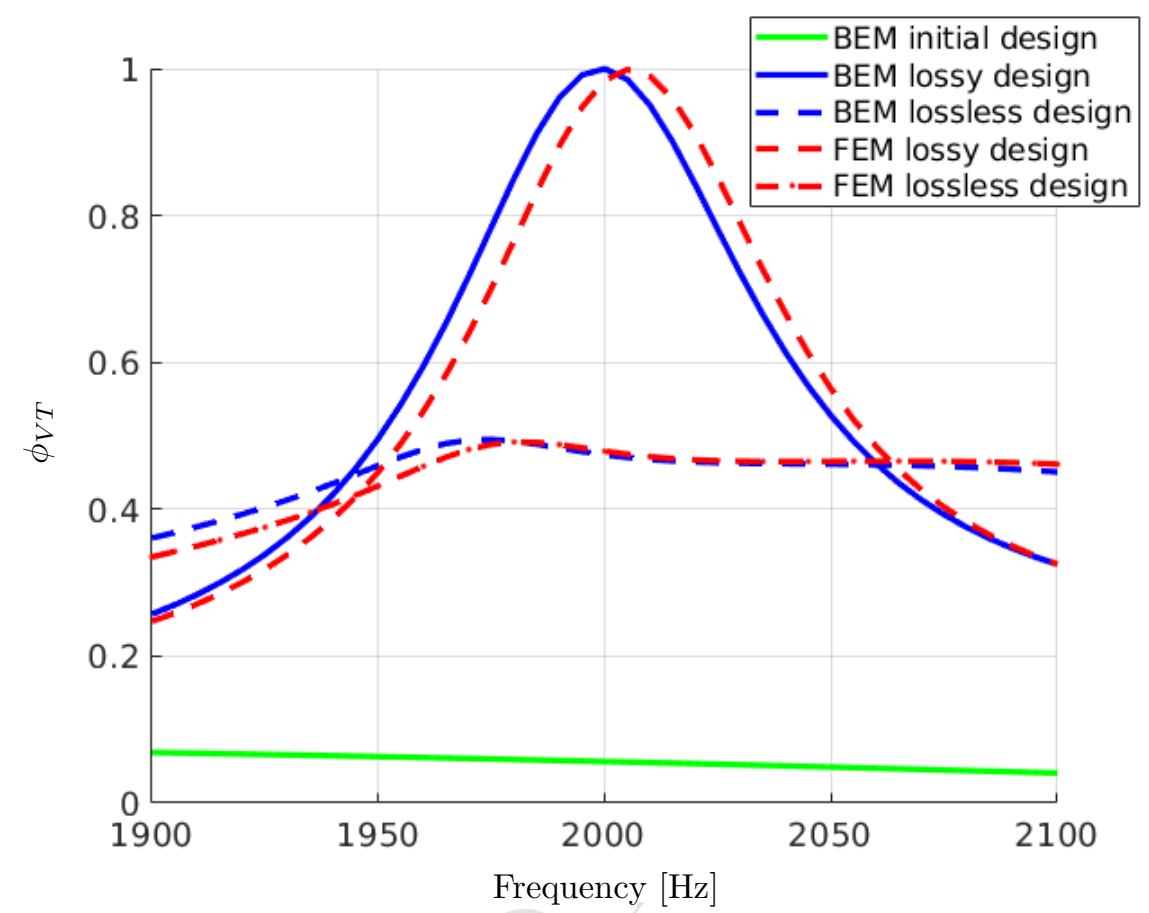

Figure 7: Absorption coefficients, calculated including losses, as a function of frequency for the initial, lossless and lossy designs. The solutions for the lossless and lossy designs are compared with corresponding lossy FEM simulations.

The absorption coefficient as a function of frequency, calculated including losses, is plotted in Fig. 7 for both initial, lossless, and lossy design. As expected, the lossy design outperforms the lossless design, yielding near-perfect absorption at the optimization frequency. However, it is worth noting that the lossless design provides an improvement as compared to the initial design. For comparison, Fig 7 includes lossy FEM calculations (red curves) of the corresponding lossless and lossy design. BEM and FEM simulations show almost the same frequency absorption response; however, a $5 \mathrm{~Hz}$ frequency shift is observed between the two methods. It is not uncommon to register differences between BEM and FEM models near resonances [38, 39, 40. Additionally, the FEM geometry might be slightly altered when compared to that of the BEM. The 
FEM simulations were performed in COMSOL Multiphysics ${ }^{\circledR}$ [4], with a geometry made up of very fine polygons, obtained from an interpolation that uses the BEM shape functions to create additional points along the boundary. This strategy destroys the quadratic geometry discretization in the FEM simulations and most likely contributes to some discrepancies. On the other hand, the FEM simulations were discretized using significantly more elements (approximately 8 million degrees of freedom).

\section{Resonators}

$\phi_{V T}=0.09$
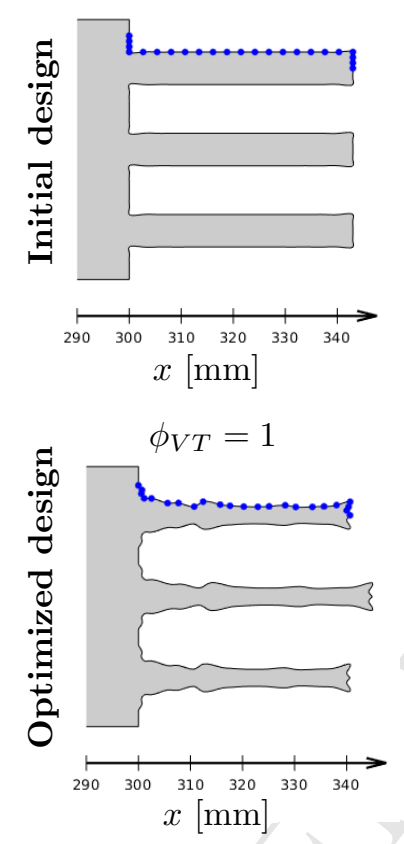

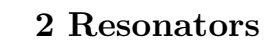

$$
\phi_{V T}=0.12
$$

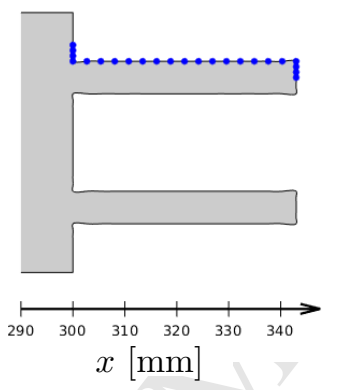

$$
\phi_{V T}=1
$$

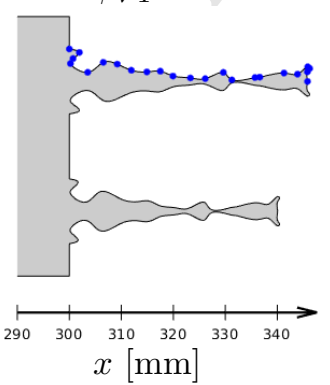

1 Resonator $\phi_{V T}=0.11$

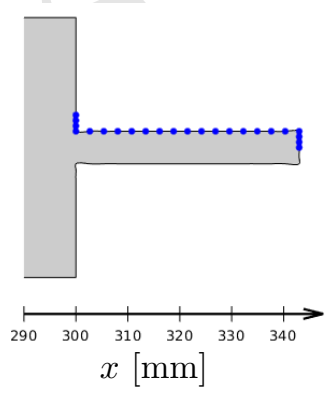

$\phi_{V T}=1$

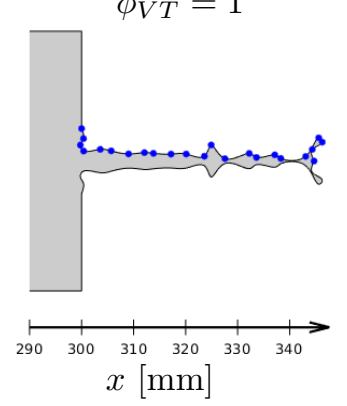

Figure 8: Shape optimization including losses using a different number of quarter-wave resonators at the impedance tube termination. The number of resonators are either three, two or one. The blue dots represent the location of the control points.

\subsubsection{The number of quarter-wave resonators}

All shape optimization examples considered so far consist of four quarterwave resonators located at the impedance tube termination. To demonstrate the applicability and generality of the shape optimization technique, Fig. 8 
contains lossy shape optimization results where the termination is parametrized with either one, two or three resonators. The figure also includes the value of the corresponding lossy objective functions, suggesting full absorption no matter the number of resonators, even in the case of only one. This shows that high absorption can be achieved without pairs of de-tuned resonators of the resonators. Again, to better understand the different damping mechanisms present in the three designs, the thermal pressure and viscous velocity are plotted in Fig 9. From this figure, it is clear that the optimizer seems to prefer the de-tuning whenever possible. When de-tuning is not a possible solution, the amount of overlapping boundary layer is dramatically increased at the end of the quarter wave-resonator, while the entrance remains relatively open. This presumably allows the acoustic wave to enter the resonator and then use the overlapping boundary layers as the main loss mechanism.

\section{Resonators}

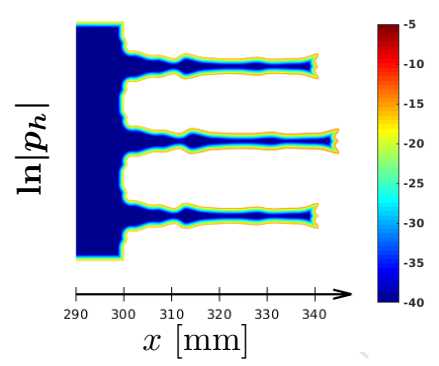

$x[\mathrm{~mm}]$

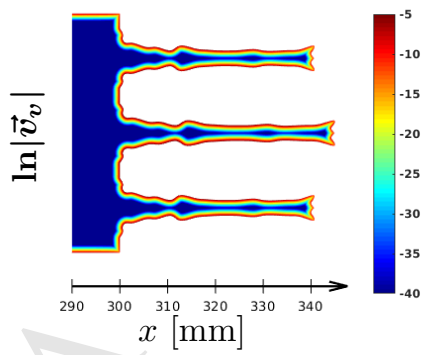

2 Resonators
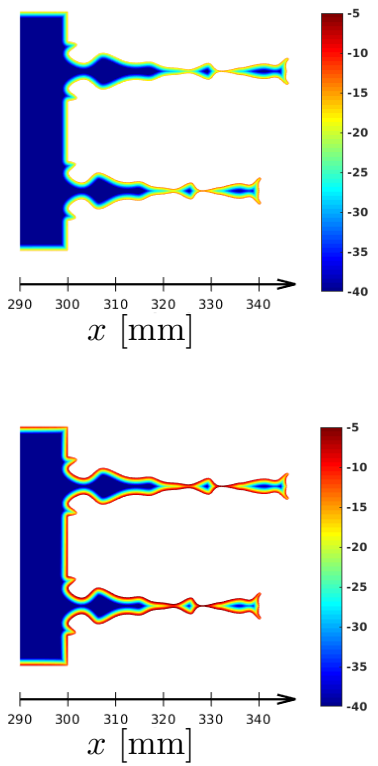

1 Resonator

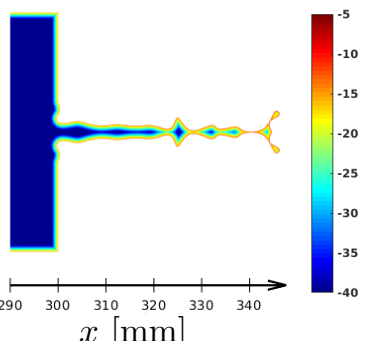

$x[\mathrm{~mm}]$
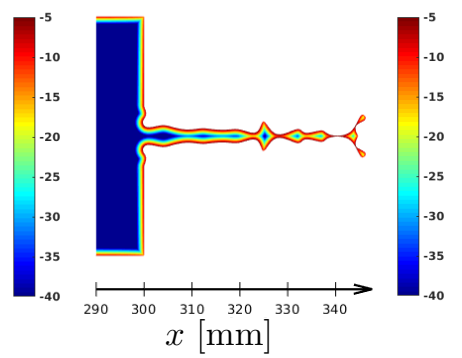

Figure 9: Domain calculations of the thermal pressure and the viscous velocity for the three shape optimized designs with a different number of quarter-wave resonators. 


\subsubsection{Number of design variables and starting guess}

Due to the non-linear and non-convex nature of the examined optimization problem, changing the number of design variables and the initial design will almost always lead to different local minima. This is demonstrated in Fig. 10. where the initial parametrization contains either less or more design variables as compared to the previously studied quarter-wave resonator parametrization. As expected, changing the initial number of design variables leads to different optimized designs, but still with near-perfect absorption. This suggests that there exists a multitude of geometric layouts, all having near-perfect absorption. Similarly, the shape of the initial parametrization can affect the optimized result.

Inspecting the optimized design in Fig. 11p and comparing it to the one in Fig. 4 (right) further motivates the hypothesis of a very flat solution space. That is, even though only a single design variable was perturbed compared to the result in Fig. 4 the optimized design is clearly different in layout while still having near-perfect absorption. a)

$$
\phi_{V T}=1
$$

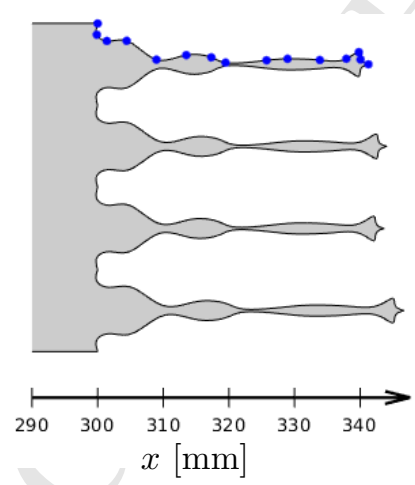

b)

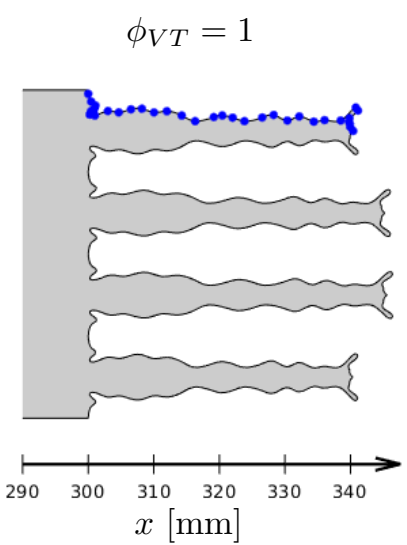

Figure 10: Shape optimization including losses of the quarter-wave resonators assuming a parametrization consisting of a) 15 control points or b) 33 control points. The blue dots represent the location of the control points after optimization. 
a)

$$
\phi_{V T}=0.06
$$

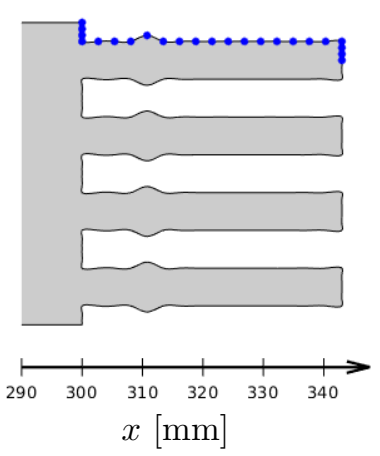

b)

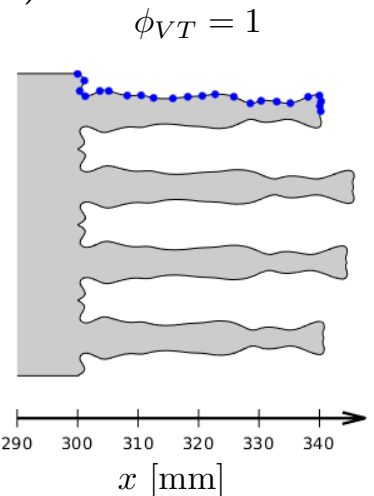

Figure 11: Shape optimization including losses of the quarter-wave resonators assuming the same parametrization as in Fig. 2 , but with an initial small perturbation of a single control point. In the figure, a) is the initial design and b) is the optimized design. The blue dots represent the location of the control points after optimization.

\subsubsection{Multi-frequency optimization of quarter-wave resonators}

Single frequency optimization limits absorption of the quarter-wave resonators to a narrow band, and in acoustic applications broadband absorption is often desired. A broader absorption response is obtained by including additional optimization frequencies as a part of the objective function. The multi-frequency objective function is defined as the sum of individual objective functions calculated at different frequencies,

$$
\phi=\sum_{i} \tilde{\phi}\left(\omega_{i}\right)
$$

where index $i$ denotes the specific optimization frequency and $\tilde{\phi}$ is the objective function evaluated at a single frequency. Fig. 12 presents multi-frequency optimization results of the quarter-wave resonators. In this case, the setup is optimized at $1950 \mathrm{~Hz}, 2000 \mathrm{~Hz}$, and $2050 \mathrm{~Hz}$. The additional optimization frequencies increase the frequency range at which sound waves are absorbed, with almost full absorption from 1900 to $2100 \mathrm{~Hz}$. The figure also includes insets with the optimized design and the corresponding pressure field magnitudes at the three optimization frequencies. Compared to the previous single frequency 


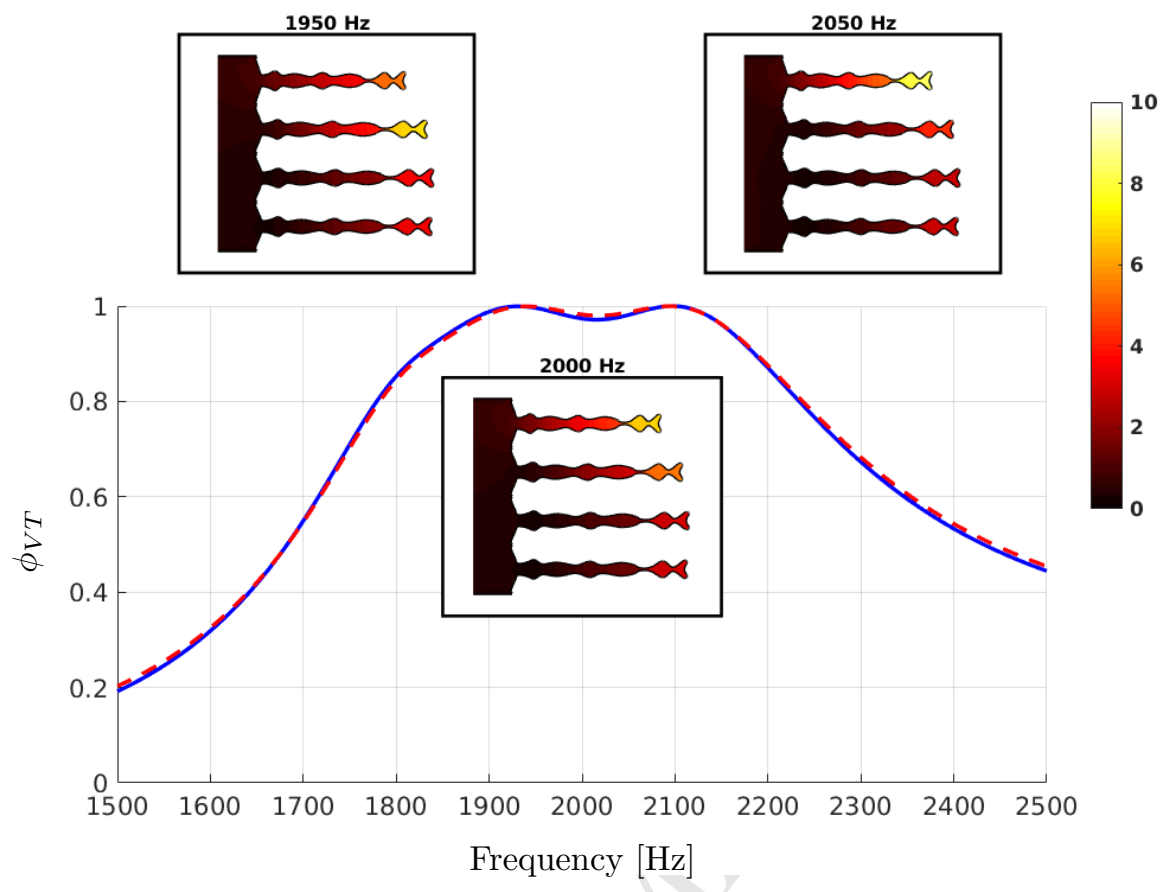

Figure 12: Multi-frequency optimization results, using the sum of the objective functions at 1950, 2000 and $2050 \mathrm{~Hz}$. Field pressure magnitudes are plotted for each optimization frequency. The solid blue and dashed red lines are BEM and FEM simulations, respectively. Both BEM and FEM simulations are calculated including viscothermal losses.

designs, the resonators have slightly narrower passages and increased variation along the quarter-wave resonator boundaries. Note also, that the behaviour of the individual quarter-wave resonators changes with frequency. At $2050 \mathrm{~Hz}$, the highest pressure magnitude is observed in the shortest resonator, whereas lower frequencies tend to excite the longer resonators. The length of the quarter-wave resonators is seen to be the primary factor that determines the operational frequency. By having different quarter-wave resonator lengths, the frequency span with high absorption is therefore increased.

\subsubsection{Bulk loss description}

A common practice when comparing experimental work with lossless simulations is to correct the simulations with an added bulk loss, e.g., using a complex 


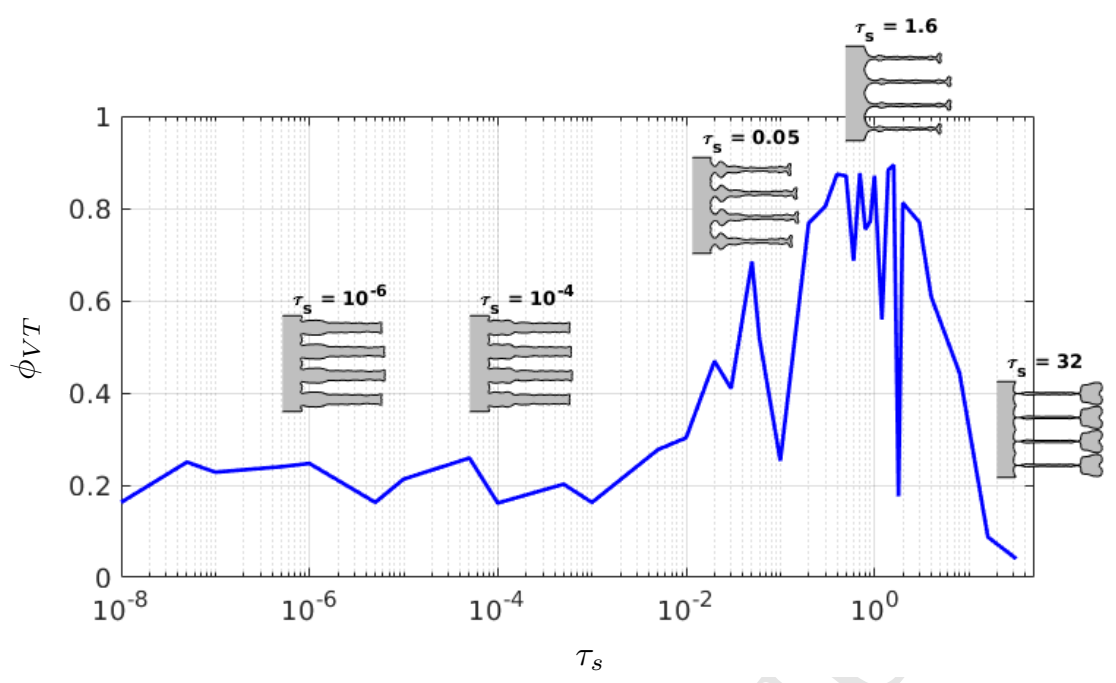

Figure 13: A series of shape optimization results of quarter-wave resonators at $2 \mathrm{kHz}$ with the acoustic losses modeled using a complex wavenumber with a tunable imaginary part. Here, $\tau_{s}$ is the value of the imaginary part of a complex wavenumber and $\phi_{V T}$ is the absorption coefficient calculated including viscothermal losses for a given design. The figure includes selected designs obtained with $\tau_{s}$ equal to $10^{-6}, 10^{-4}, 0.05,1.6$ and 32 .

wavenumber with a controllable imaginary part. However, no general procedure exists to estimate the magnitude of the imaginary part, and it is typically problem dependent [42. It is therefore interesting to investigate how varying the imaginary part of the wavenumber affects the optimization of the quarterwave resonators. This is accomplished by defining the complex wavenumber as $k_{B}=k_{L L}-i \tau_{s}$, where $\tau_{s}$ is the tunable parameter and $k_{L L}$ is the isentropic wavenumber. In Fig. 13 , optimized designs are presented using different values of $\tau_{s}$ and compared to the actual absorption coefficient, calculated including viscothermal losses. It is seen how certain values of $\tau_{s}$ result in high absorption coefficients above $80 \%$. However, none of the used values for $\tau_{s}$ results in a design with near-perfect absorption. It should also be noted that only a limited amount of simulations with different values of $\tau_{s}$ were performed and that there might exist values of $\tau_{s}$ resulting in higher absorption coefficients. In the range $\tau_{s} \in\{0.01,3\}$, the absorption coefficients are very sensitive to the 


\section{Lossless optimization}
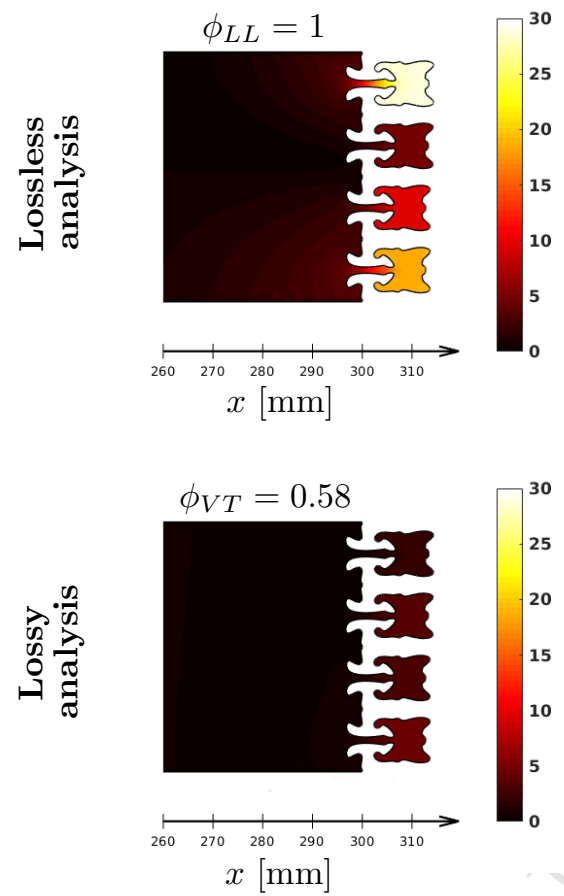

Lossy optimization
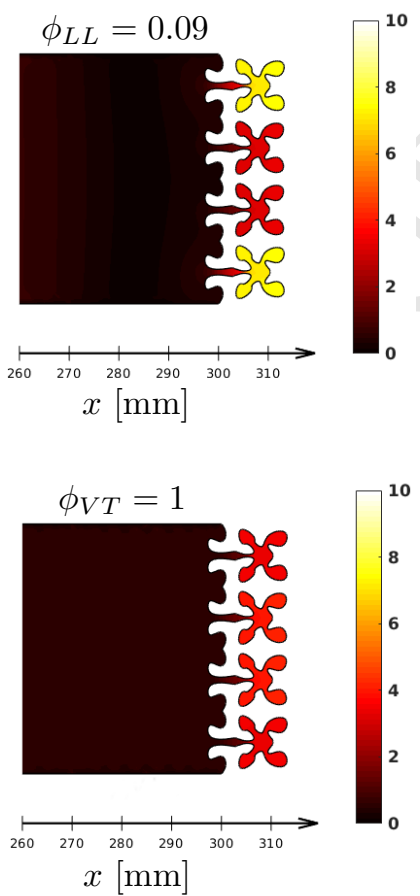

Figure 14: Optimization results of Helmholtz resonators located at the impedance tube termination. The designs are based on either a lossless or a lossy assumption. The field variables are the pressure magnitudes in Pascal

choice of $\tau_{s}$, making it difficult to choose an appropriate value for this particular example. Fig. 13 also depicts inserts with selected designs. It is noteworthy to highlight the design corresponding to $\tau_{s}=0.05$ because it is very similar to the single frequency lossy quarter-wave resonator design, although its performance is inferior.

\subsection{Helmholtz resonators}

In this section, the impedance tube termination is initially parametrized forming four equally sized Helmholtz resonators as described in Fig $2 \mathrm{~b}$. The neck and cavity dimensions of the initial shape of the resonators yields an operational 
frequency near $2 \mathrm{kHz}$. The Helmholtz resonators are, as in the case of the quarter-wave resonators, optimized under both lossless and lossy conditions. The optimization is carried out at $2 \mathrm{kHz}$. Fig. 14 contains the resulting lossless and lossy designs which are overlaid with acoustic pressure magnitude field calculations of both the lossless and lossy solutions. Similar to the quarterwave optimization results, the performance of the lossless design is degraded when introducing losses. When calculated without losses, the lossless design contains varying high pressure amplitudes between the individual cavities of the Helmholtz resonators, which are de-tuned in a similar way to the quarterwave resonators. However, the pressure differences and de-tuning disappears when calculated with losses.

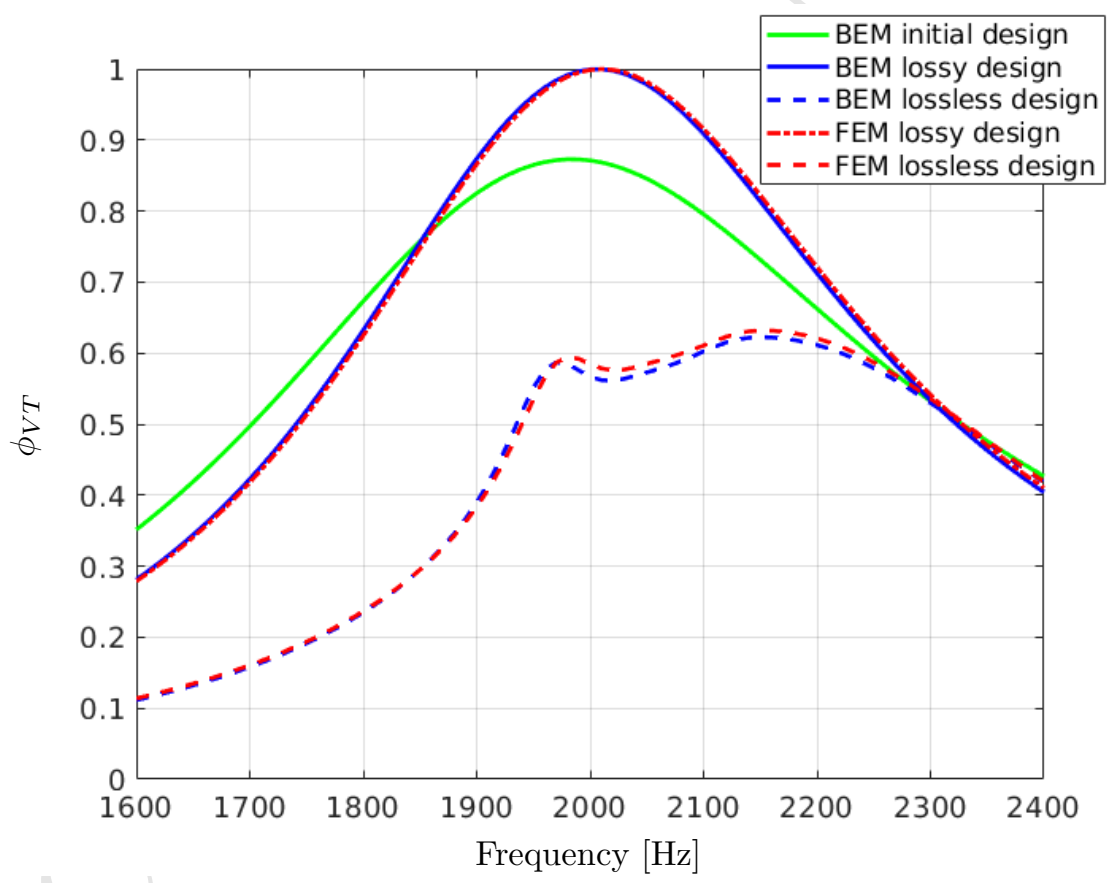

Figure 15: The absorption coefficient as a function of frequency for the Helmholtz resonators, shape optimized under lossless and lossy conditions. Both BEM and FEM simulations are calculated including viscothermal losses.

In the case of optimization including losses, the design outcome deviates far 


\begin{tabular}{|c|c|c|c|}
\cline { 2 - 4 } \multicolumn{1}{c|}{} & Lossless design & Lossy design & Characteristic impedance \\
\hline$\left|Z_{\text {avg }}\right|\left[\frac{\text { Pa.s }}{\mathbf{m}}\right]$ & 261.66 & 412.86 & 413.09 \\
\hline$\angle Z_{\text {avg }}[$ rad $]$ & 0.59 & -0.02 & 0 \\
\hline
\end{tabular}

Table 1: The average impedance and phase near the impedance tube termination compared with the characteristic impedance.

from the classical Helmholtz resonator shape, with the cavity best described as a four-leaf clover. Usually, the neck of Helmholtz resonators is the primary source of viscothermal dissipation. In this case, however, it is believed that the clover shape contributes to increased damping, with closer boundaries. Whether the lossy design can still be considered a Helmholtz resonator is unclear, but it does include a neck and a slightly unconventionally shaped cavity. While the overall shape between the lossless and lossy design deviates, the neck shape at the entrance of the Helmholtz resonators is very similar.

The absorption coefficient as a function of frequency is plotted in Fig. 15 Interestingly, optimized designs based on a lossless assumption produce poorer designs when compared to the initial guess, indicating that numerical damping alone is not sufficient in this case. On the other hand, including viscothermal losses during optimization results in an improved design, with near-perfect absorption at the optimization frequency. The figure includes viscothermal FEM calculations, with conclusions similar to those in Section 5.1 .

Another relevant performance criterion besides the absorption coefficient is the impedance at the termination. For a perfect absorber, with an absorption coefficient equal to 1 , an impedance close to the characteristic impedance is expected. Calculations of the average impedance magnitude and phase across the terminations are given in Table 1 and compared to the characteristic impedance corresponding to the ambient conditions assumed in the simulations. While the lossy design matches the average characteristic impedance very well, it should, however, be noted that the impedance across the termination varies slightly across the cross-section. For the lossless design, it is obvious that the impedance 
criterion is not fulfilled. Since the aim of this paper is on viscothermal losses, we do not investigate the impedance mismatch in the lossless setting further.

\section{Discussion}

This paper presents an acoustic BEM shape optimization technique including viscous and thermal dissipation. It is successfully applied to maximize the absorption coefficient of two classical two-dimensional acoustic resonators located at an impedance tube termination. The potential problems when neglecting acoustic dissipation during optimization are investigated. In both the case of quarter-wave and Helmholtz resonator simulations, the designs yield significantly better performance if optimized upon a lossy assumption. In all lossy designs, the final resonator designs yield near-perfect absorption at the desired frequencies. It is also shown, that the proposed method is capable of achieving near-perfect absorption when solved with varying number of the design variables and different starting configurations. On the other hand, lossless optimization has proven to develop poorly performing resonators. However, the outcome depends on the resonator type. The quarter-wave resonators, optimized on a lossless assumption, deliver an improved design over the initial starting guess. This is mainly due to the existence of numerical damping in BEM. In the case of the Helmholtz resonators, optimization without viscothermal losses creates a poorer design when compared to the initial resonator shape. Additionally, it is examined whether a simplified bulk loss description of losses can be used to improve the optimization result. It turns out to be difficult to estimate an appropriate value of the loss parameter $\tau_{s}$. Moreover, none of the studied values of $\tau_{s}$ has resulted in near-perfect absorption when analysed using the viscothermal model.

The combination of viscothermal BEM and shape optimization is a promising tool and can be applied to the development of a range of acoustic applications where viscothermal losses are relevant. The current implementation is however limited to computationally light problems, due to the utilization of an 
inefficient FD sensitivity estimation. Future work should therefore investigate methods for obtaining more accurate and efficient sensitivities, i.e., development and implementation of an adjoint method. This would allow for the study of three-dimensional test cases suitable for experimental validation. In general, experimental realization of optimized acoustic designs will require the inclusion of manufacturing constraints and robustness measures. The proposed technique includes constraints that might be applicable, but not necessarily sufficient for this purpose, i.e., the curvature and distance constraints. Additionally, the proposed shape optimization technique can be extended to account for mechanical coupling which often plays an important role in small micro-acoustic devices. Finally, it would be interesting to pursue alternative objectives or multi-objectives such as impedance and total energy loss.

\section{Appendix A. Sparse assembly of viscous and thermal matrices}

The assembly speed of the viscous and thermal matrices is improved by selectively performing integration when the distance between the collocation point and the element is less than twenty times the boundary layer thickness. The viscous and thermal Green's functions are defined by

$$
G\left(k_{j} R\right)=\frac{1}{2 \pi} K_{0}\left(i k_{j} R\right)
$$

where $K_{0}$ is the modified Bessel function of second kind of zero order and the index $j$ denotes either the acoustic, thermal or viscous wavenumber. In general, the thermal and viscous boundary layer thicknesses, $\delta_{h}$ and $\delta_{v}$, will be frequency dependent following the relation, 1 ]

$$
\begin{aligned}
\delta_{h} & =\sqrt{\frac{2 \lambda}{\rho_{0} \omega C_{p}}} \\
\delta_{v} & =\sqrt{\frac{2 \mu}{\rho_{0} \omega}}
\end{aligned}
$$

where $\lambda$ is the thermal conductivity, $C_{p}$ is the specific heat at constant temperature, $\rho_{0}$ is the static density, $\mu$ is the shear viscosity and $\omega$ is the angular 
frequency. To illustrate the dimension of the truncation error this approach

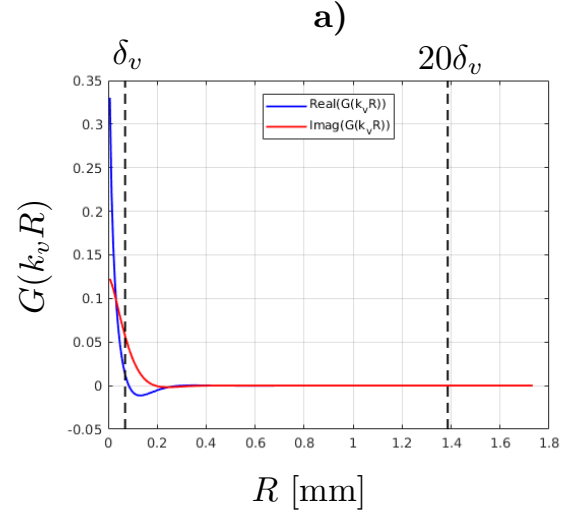

might introduce, the fundamental solution is plotted in Figure A.1 using both the viscous and thermal wavenumber calculated at $1 \mathrm{kHz}$. It is observed that the magnitude of the fundamental solution is very small at the truncation location, approximately equal to $10^{-10}$, which is around nine orders of magnitude smaller as compared with the solution near the boundary. Therefore, the truncation approach should not contribute with any significant errors.

a)

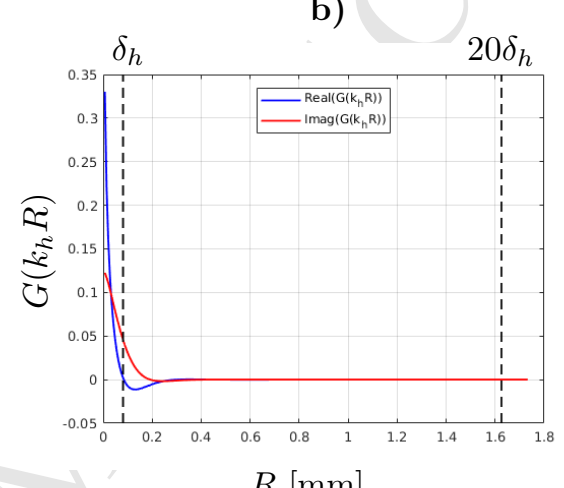

Figure A.1: The fundamental solution calculated at $1 \mathrm{kHz}$ using either a) the viscous wavenumber or b) the thermal wavenumber. Here, $\delta_{v}$ and $\delta_{h}$ are the boundary layer thicknesses of the viscous and the thermal boundary layers, respectively[1]. The black striped lines correspond to one and twenty times the boundary layer thickness.

\section{Appendix B. Finite difference step length}

Shape optimization is performed using the default step length in fmincon which is equal to $\Delta v_{i}=\sqrt{\mathrm{eps}} \approx 1.5 \cdot 10^{-8}$, where eps denotes the relative accuracy, for double-precision IEEE floating-point representation. The finite difference step length is important for the accuracy of the calculated sensitivities. To ensure that the default step length is accurate for the studied cases, the finite difference sensitivities are plotted in B.1 for all the design variables assuming different step lengths. In general, the goal is a step length that lies in the region where the gradient can be considered constant. The investigation shows that, for this particular problem, it is safe to use the default step length in fmincon. 


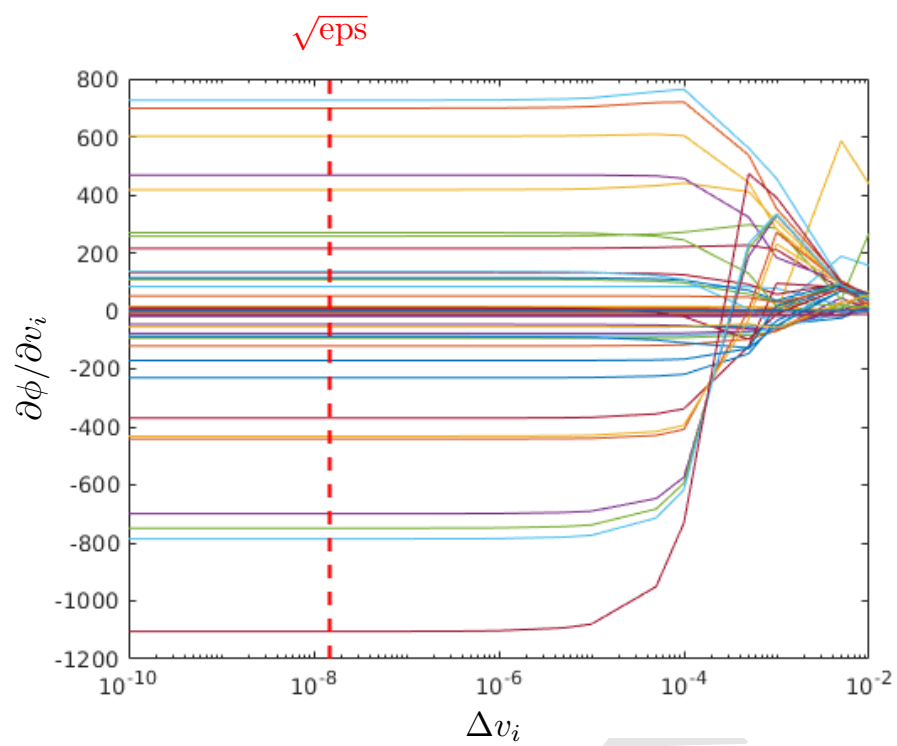

Figure B.1: The finite difference gradients of the objective function, calculated at different step sizes for all the design variables. The initial geometry corresponds to the $5^{\text {th }}$ iteration in Figure 6 of the quarter-wave resonators optimized including losses. The red striped line denotes the location of the default step length in fmincon.

\section{References}

[1] P. M. Morse, K. U. Ingard, Theoretical Acoustics, McGraw-Hill, Princeton, 1968.

[2] A. D. Pierce, Acoustics. An introduction to its principles and applications (Ch. 10), McGraw Hill, New York, 1981.

[3] R. Kampinga, Viscothermal acoustics using finite elements analysis tools for engineers, Ph.D. thesis, University of Twente, Enschede (2010).

[4] R. Christensen, Acoustic modeling of hearing aid components, Ph.D. thesis, University of Southern Denmark (2010).

[5] D. Homentcovschi, R. N. Miles, P. Loeppert, A. J. Zuckerwar, A microacoustic analysis including viscosity and thermal conductivity to model 
the effect of the protective cap on the acoustic response of mems mi-

crophone, Microsyst Technol. 20 (2) (2014) 265-275. doi:10.1007/ s00542-013-1800-5.

[6] V. Cutanda Henríquez, P. M. Juhl, Modelling measurement microphones using bem with visco-thermal losses, Proceedings of the Joint Baltic-Nordic acoustic Meeting (BNAM), Odense (Denmark), 2012.

[7] R. Christensen, U. Skov, Compression driver simulation incl. vibroacoustic, viscothermal \& porous acoustics, in: Proceedings from the COMSOL conference in Stuttgart (Germany), 2011.

q [8] R. Christensen, U. Skov, Simulation of a 4" compression driver using a fully

1. coupled vibroacoustic finite element analysis including viscous and thermal losses, in: Audio Engineering Society Convention 132, 2012.

URL http://www . aes .org/e-lib/browse.cfm?elib=16310

${ }_{470}$ [9] M. Berggren, A. Bernland, D. Noreland, Acoustic boundary layers as boundary conditions, Journal of Computational Physics 371 (2018) 633650. doi:10.1016/j.jcp.2018.06.005.

[10] S. A. Cummer, J. Christensen, A. Alù, Controlling sound with acoustic [ metamaterials, Nature reviews materials 1 (2016) 1-13. doi:doi:10.1038/ $475 \quad$ natrevmats.2016.1.

[11] R. E. Christensen, O. Sigmund, Exprimental validation of systematically designed acoustic hyperbolic meta material slab exibiting refraction, Appl. Phys. Lett. 109 (2016) 101905. doi:10.1063/1.4962441.

[12] V. C. Henríquez, V. García-Chocano, J. Sánchez-Dehesa, Viscothermal losses in double-negative acoustic metamaterial, Physical Review Applied 8 (12) (2017) 014029. doi:10.1103/PhysRevApplied.8.014029.

[13] V. C. Henríquez, P. Andersen, J. S. Jensen, P. M. Juhl, J. Sánchez-Dehesa, A numerical model of an acoustic metamaterial using the Boundary Ele- 
ment Method including viscous and thermal losses, Journal of Computational Acoustics 25 (4) (2017) 1750006. doi:10.1142/S0218396X17500060

[14] M. Molerón, M. Serra-Garcia, C. Daraio, Visco-thermal effects in acoustic metamaterials: from total transmission to total reflection and high absorption, New J. Phys. 18 (2016) 033003. doi:10.1088/1367-2630/18/3/ 033003 .

[15] N. Jiménez, V. Romero-García, P. Pagneux, J. Groby, Rainbow-trapping absorbers: Broadband, perfect and asymmetric sound absorption by subwavelength panels for transmission problems, Scientific Reports 7 (1) (2017) 13595. doi:10.1038/s41598-017-13706-4.

[16] J. Carbajo, J. Ramis, Godinho, J. Amado-Mendes, P., J. Alba, A finite element model of perforated panel absorber including viscothermal effects,

口 Journal of Applied Acoustics 90 (2014) 1-8. doi:10.1016/j.apacoust. 2014.10 .013

[17] A. I. Komkin, M. A. Mirov, A. I. Bykov, Sound absorption by a helmholtz resonator, Acoustical Physics 63 (4) (2017) 385-392. doi:10.1134/ S1063771017030071.

[18] R. Udawalpola, E. Wasbro, M. Berggren, Optimization of a variable mouth horn, Int. J. Numer. Meth. Engng 85 (2010) 591-606. doi:10.1002/nme. 2982.

[19] E. Wadbro, M. Berggren, Topology optimization of an acoustic horn, Comput. Methods Appl. Mech. Engrg. 196 (2006) 420-436. doi:10.1016/j. cma.2006.05.005.

[20] R. Udawalpola, E. Wasbro, M. Berggren, Optimization of an acoustic horn with respect to efficientcy and directivity, Int. J. Numer. Meth. Engng 73 (2007) 1571-1606. doi:10.1002/nme.2132. 
[21] R. Christensen, Topology optimization of thermoviscous acoustics in tubes and slits with hearing aid applications, Proceedings of the COMSOL Conference in Rotterdam.

[22] V. Cutanda Henríquez, P. Risby Andersen, A three-dimensional acoustic boundary element method formulation with viscous and thermal losses based on shape function derivatives, Journal of Computational and Theoretical Acoustics 26 (3) (2018) 1859939. doi:10.1142/ S2591728518500391.

[23] M. Malinen, M. Lyly, P. Raback, A. Karkainen, L. Karkkainen, A Finite Element Method for the modeling of thermo-viscous effects in acoustics, Proceedings of the 4th European Congress on Computational Methods in Applied and Engineering ECCOMAS, Jyvaskyla (Finland).

[24] M. Bruneau, P. Herzog, J. Kergomard, J. D. Polack, General formulation of the dispersion equation in bounded visco-thermal fluid, and application

4. to some simple geometries, Wave Motion 11 (1989) 441-451doi:10.1016/ 0165-2125(89) 90018-8.

[25] V. Cutanda Henríquez, P. M. Juhl, An axisymmetric boundary element formulation of sound wave propagation in fluids including viscous and thermal losses, Journal of the Acoustical Society of America 134 (5) (2013) 3409. doi:10.1121/1.4823840.

[26] V. Cutanda Henríquez, P. M. Juhl, OpenBEM - an open source Boundary Element Method software in acoustics,, Proceedings of the 39th International Congress on Noise Control Engineering, Inter-noise, Lisbon (Portugal).

[27] ISO 10534-2:1998, Acoustics - Determination of sound absorption coefficient and impedance in impedance tubes - Part 2: Transfer-function method, Standard, International Organization for Standardization (1998). 
[28] MATLAB optimization toolbox, the MathWorks, Natick, MA, USA (2017b).

[29] M. P. Bendøe, O. Sigmund, Topology Optimization Theory, Methods, and Applications, Springer, 2004.

[30] A. J. Canós, Fast and robust self-intersections, https :

1 / / se . mathworks . com / matlabcentral / fileexchange / 13351-fast-and-robust-self-intersections accessed: 2018-2510.

[31] I. N. Bronshtein, K. A. Semendyayev, G. Musiol, H. Muehlig, Handbook of mathematics, fifth edition, Springer Berlin Heidelberg, 2007, p. 229.

[32] L. Chen, L. Liu, W. Zhao, H. Chen, 2D acoustic design sensitivity analysis based on adjoint variable method using different types of boundary elements, Acoust. Aust. 44 (2016) 343-357. doi:10.1007/ s40857-016-0065-4.

[33] J. Fahnline, Numerical difficulties with boundary element solutions of interior acoustic problems, Journal of Sound and Vibration 319 (2008) 10831096. doi:10.1016/j.jsv.2008.06.040

[34] S. Marburg, Numerical damping in the acoustic boundary element method, Acta Acustica United with Acustica 102 (2018) 1550016. doi:10.3813/ AAA.918958.

[35] S. Marburg, A pollution effect in the boundary element mmethod for acoustic problems, Journal of Computaional Acoustics 26 (18) (2018) 1850018. doi:10.1142/S0218396X18500182.

[36] H. Ryoo, W. Jeon, Dual-frequency sound-absorbing metasurface based on viscothermal effects with frequency dependence, Journal of Applied Physics 123 (2018) 115110. doi:10.1063/1.5017540. 
[37] J. Li, W. Wang, Y. Xie, B. Popa, S. A. Cummer, A sound absorbing metasurface with coupled resonators, Appl. Phys. Lett. 109 (2016) 091908. doi:10.1063/1.4961671.

[38] N. Aage, V. Egede Johansen, Topology optimization of microwave waveguide filters, International Journal for Numerical Methods in Engineering 112 (3) (2017) 283-300. doi:10.1002/nme.5551.

[39] N. Aage, Mortensen, N. A., O. Sigmund, Topology optimization of metallic devices for microwave applications, International Journal for Numerical Methods in Engineering 83 (2) (2010) 228-248. doi:10.1002/nme.2837.

[40] A. R. Diaz, O. Sigmund, A topology optimization method for design of negative permeability metamaterials, International Journal for $\mathrm{Nu}-$ merical Methods in Engineering 41 (2) (2010) 163-177. doi:10.1007/ s00158-009-0416-y.

[41] COMSOL Multiphysics Reference Manual, version 5.2, COMSOL, Inc, www.comsol.com.

[42] R. E. Christensen, E. Fernandez-Grande, Experimental validation of a topology optimized acoustic cavity, J. Acoust. Soc. Am. 138 (2015) 34703474. doi:10.1121/1.4936905. 